\title{
Role of Chest Ultrasound in Detection of the Cause of Pleural Effusion and Guidance for Thoracentesis
}

\author{
H.E.EL-Sheikh ${ }^{1}$, T.S.Essawy ${ }^{2}$, H.M.khater ${ }^{1}$ and M.A.EL-Gazzar ${ }^{1}$ \\ ${ }^{1}$ Radiodiagnosis Dept., Faculty of Medicine, Benha Univ., Benha, Egypt \\ ${ }^{2}$ Chest Diseases Dept., Faculty of Medicine, Benha Univ., Benha, Egypt \\ E-Mail:maram11@gmail.com
}

\begin{abstract}
Pleural emission can result from various conditions, for example, congestive cardiovascular breakdown, pneumonia, malignant growth, liver cirrhosis, and kidney illness. The attributes of the liquid rely upon the fundamental pathophysiologic system. The liquid can be transudate, nonpurulent exudate, discharge, blood, or chyle. Imaging examines are important in recognizing and overseeing pleural emissions yet not in precisely portraying the biochemical idea of the liquid. Ultrasound is more delicate than actual test and chest radiography to distinguish and describe pleural liquid, and dodges many negative parts of electronic tomography (CT). Ultrasound can be utilized to survey pleural liquid volume and character, uncovering conceivable fundamental pathologies and controlling administration.. Objective. To survey the utilization of ultrasound for the location of pleural emissions and direction of the thoracentesis technique. Pleural goal portrays a technique whereby pleural liquid or air might be suctioned by means of a framework embedded briefly into the pleural space. This might be for demonstrative purposes (normally eliminating 20-50 ml liquid) or restorative to soothe manifestations. In the writing it is varyingly called thoracocentesis, thoracentesis or pleural yearning.
\end{abstract}

Keywords: Pleural effusion, Lung, Ultrasonography, Catheters, Critical care.

\section{Introduction}

A pleural emanation is a development of liquid in the pleural space, a region between the layers of tissue that line the lungs and the chest divider. It might likewise be alluded to as radiation or aspiratory effusion [1].

Patients might be asymptomatic however frequently present with dyspnea, chest torment, hack, or different signs or manifestations of hidden illness. Dyspnea is an aftereffect of modified diaphragmatic and chest divider mechanics and pressure of the lung. Pleuritic chest torment shows illness contribution of the parietal pleura [2]

There are likewise confounded and straightforward pleural emanations. Straightforward pleural emissions contain liquid without indications of disease or irritation. They're substantially less prone to cause lasting lung issues. Convoluted pleural emanations, be that as it may, contain liquid with huge disease or aggravation. They require brief treatment that much of the time incorporates chest drainage [3].

It was felt that ultrasound couldn't be utilized to survey the chest. The primary organs of the chest contain air, which doesn't communicate ultrasound appropriately, related with the way that the ribs block it. Ultrasonography has, in any case, become a truly significant asset in the assessment of the strange chest, where fluid and strong densities are intervened between the chest divider and the lung, making it a superb methods for sending sound waves, making this strategy was stretched out to a progression of morbidities [4]

Convenient ultrasonography gadgets are utilized at the bedside to assess pleural anomalies and to direct thoracentesis and related systems, for example, pleural seepage, catheter arrangement, and needle desire biopsy of pleural or sub pleural lung masses [5].
Numerous signs for the utilization of ultrasonography past the representation of the pleura and related conditions (counting emanations, thickening and pneumothorax) have been approved over the most recent couple of many years. These incorporate the evaluation of diaphragmatic brokenness, aspiratory solidification, interstitial conditions, pneumonic embolism, and pneumonic and mediastinal tumors, if they adjoin the pleura [6].

The procedure is especially fit to bedside use in the emergency unit, imperfect radiography may veil or copy clinically critical anomalies and where separation of pleural from parenchymal changes can be testing. Ultrasound permits the location of modest quantities of pleural locular liquid, with positive distinguishing proof of sums as little as 3-5 mL, that can't be recognized by radiographs, which is just equipped for identifying volumes over $50 \mathrm{~mL}$ of liquid [7].

Brief conclusion of the presence and nature of pleural radiation is essential to assess the best remedial decision (diuretics, obtrusive methodology). The utilization of thoracic ultrasound (TUS), a safe and non-obtrusive bedside technique with great precision, can assist clinicians with envisioning the emanation and furthermore to recognize various sorts (exudative, transudative empyema, hemothorax) [8] .

Lung imaging in basically sick patients is generally performed either by bedside CXR or thoracic CT, yet the two strategies have constraints which compel their value. Ultrasound has very comparable exhibitions to CT. These days, bedside thoracic ultrasound is progressively utilized in fundamentally sick patients [9].

Thoracentesis is a technique of goal of liquid from the pleural space by percutaneous addition of a needle 
through the chest divider with or without the inclusion of a catheter [10].

The point of this investigation is to assess the part of chest ultrasound in recognition of the reason for pleural emanation and direction for thoracentesis.

\section{Patients and methods}

This study was carried out on 4. patients with pleural effusions from those admitted in radiology department of Benha university hospitals between May 2019 to June 2020 .

\section{Inclusion criteria}

- Any adult Patient with chest manifestations suspecting to have pleural effusion like dyspnea and chest pain.

- Patients with previous history of pleural effusions.

- Patients confirmed to have pleural effusion.

\section{Exclusion criteria}

- Patients on mechanical ventilation.

- Patients below 18 years old.

- In cooperative patients.

Carful history taking and chest examination with revision of all data that the patient had are all necessary to be done at first. then performing chest ultrasound for evaluation of the pleural effusion as regard; amount ,characters and if accessible to perform thoracentesis when the patient accept.

The following was done when required:

-Pleural aspiration from the effusion
-Pleural biopsy from any suspicious lesion.

-Laboratory analysis of the specimens.

-Data collection and recording.

\section{Statistical analysis}

The statistical analysis was conducted using the Software, Statistical Package for Social Science, (SPSSInc. Released 2009- PASW Statistics for Windows Version 21.0. Chicago: SPSS Inc.) The collected data were summarized in terms of mean \pm Standard Deviation (SD) and range (minimum maximum) for quantitative data and frequency and percentage for qualitative data. Comparisons between proportions were carried out using the Chi-square test $\left(\chi^{2}\right)$ when appropriate) and the Fisher Exact Test (FET) to compare proportions as appropriate. Cohen's kappa coefficient $(\kappa)$ statistic used to measure inter-rater reliability (agreement) for qualitative (categorical) items. After the calculation of each of the test statistics, the corresponding distribution tables were consulted to get the "P" (probability value). Statistical significance was accepted at $\mathrm{P}$ value $<0.05(\mathrm{SS})$. A $\mathrm{P}$ value $<0.001$ was considered highly significant (HS) while a $\mathrm{P}$ value $>0.05$ was considered non-significant (NS).

\section{Results}

Forty patients were assessed in this study. Sixty five percent of them were males and $35 \%$ were females. The mean age of the studied group was $43.8 \pm$ 13.2 and the age range between 26 and 76 years Table (1).

Table (1) Demographic characteristics of the studied group.

\begin{tabular}{|c|c|c|c|}
\hline \multirow[t]{2}{*}{ Variable } & & \multicolumn{2}{|c|}{$\begin{array}{l}\text { Frequency } \\
(n=40)\end{array}$} \\
\hline & & No. & $\%$ \\
\hline Sex & Male & 26 & 65 \\
\hline & Female & 14 & 35 \\
\hline Age & $\begin{array}{l}\text { Mean } \pm \mathrm{SD}(\text { Range }) \\
43.8 \pm 13.2(26-76)\end{array}$ & & \\
\hline
\end{tabular}

$50 \%$ of the studied group had left-sided pleural effusion, $40 \%$ had right-sided pleural effusion and $10 \%$ showed bilateral effusion. Marked pleural effusion has been dedicated in $50 \%$ of examined patients compared to $5 \%$ of patients who showed minimal effusion, $20 \%$ showed mild effusion and $20 \%$ moderate effusion. Encysted effusion has been found in $5 \%$ of examined patients Fig (1).

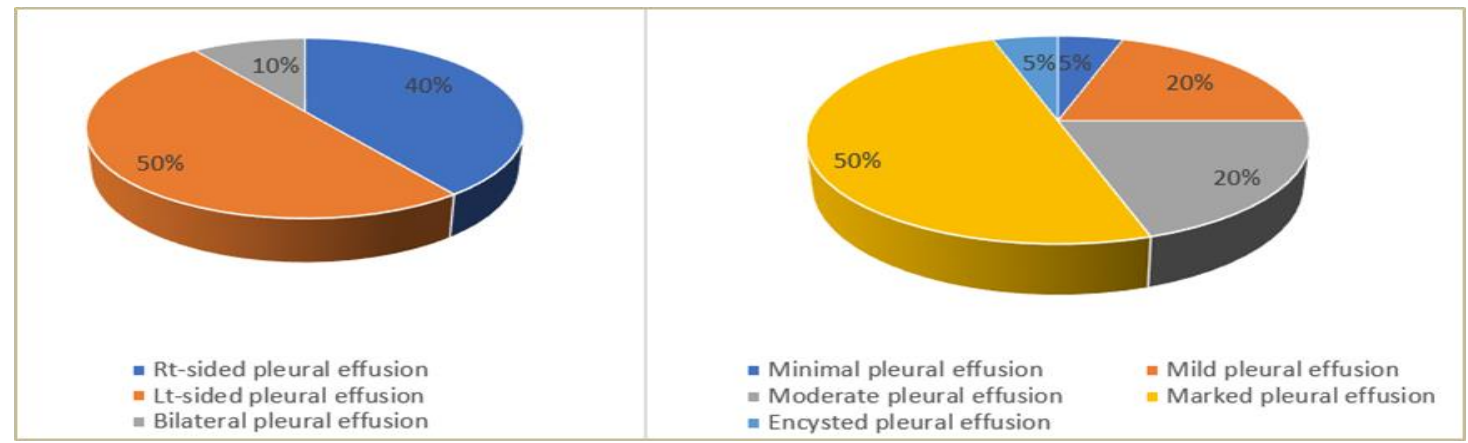

Fig (1) Pie chart shows frequency distribution of the studied patients as regard side and volume of pleural effusion. 
Very strong highly statistically significant correlation between US and Lab findings regarding in detection of the cause of pleural effusion in the studied group. $(\mathrm{P}<0.001)$.

\begin{tabular}{lccc}
\hline Variable Statistic & $\phi \mathbf{c}$ & CC & P value \\
\cline { 1 - 2 } (nominal by nominal) & 0.67 & 0.80 & $<0.001$ \\
\hline
\end{tabular}

$\phi c=$ Cramer's V coefficient $\quad \mathrm{CC}=$ Contingency coefficient.

Table (6) Correlation between Sonographic findings in relation of Lab. Diagnosis in the studied group.

$\mathrm{T}$ the sensitivity of US in diagnosis of transudate effusion was $75 \%$ and specificity $93.7 \%$, the positive predictive value was $75 \%$ and the negative predictive value was $93.7 \%$. the total accuracy was $90 \%$.

\begin{tabular}{|c|c|c|c|c|}
\hline Lab & US & positive & negative & Total \\
\hline Positive & & 6 & 2 & 8 \\
\hline Negative & & 2 & 30 & 32 \\
\hline Total & & 8 & 32 & 40 \\
\hline
\end{tabular}

Test of significant is Chi square $(\mathrm{X} 2)=18.9$

$P$ value $<0.001$ (highly significant)

Table (7) Predictability of US in diagnosis of transudate effusion:The sensitivity of US in diagnosis of parapneumonic effusion was $50 \%$ and specificity $75 \%$, the positive predictive value was $33.3 \%$ and the negative predictive value was $85.7 \%$. the total accuracy was $70 \%$.

\begin{tabular}{rcccc}
\hline Lab & US & positive & negative & Total \\
\hline Positive & $\varepsilon$ & $\wedge$ & $1 r$ \\
Negative & $\varepsilon$ & $r \varepsilon$ & $r \wedge$ \\
Total & $\wedge$ & $r r$ & $\varepsilon \cdot$ \\
\hline
\end{tabular}

Test of significant is Chi square $(\mathrm{X} 2)=1.9$

$\mathrm{P}$ value $=0.16$ (non-significant)

Table (8) Predictability of US in diagnosis of parapneumonic effusion.

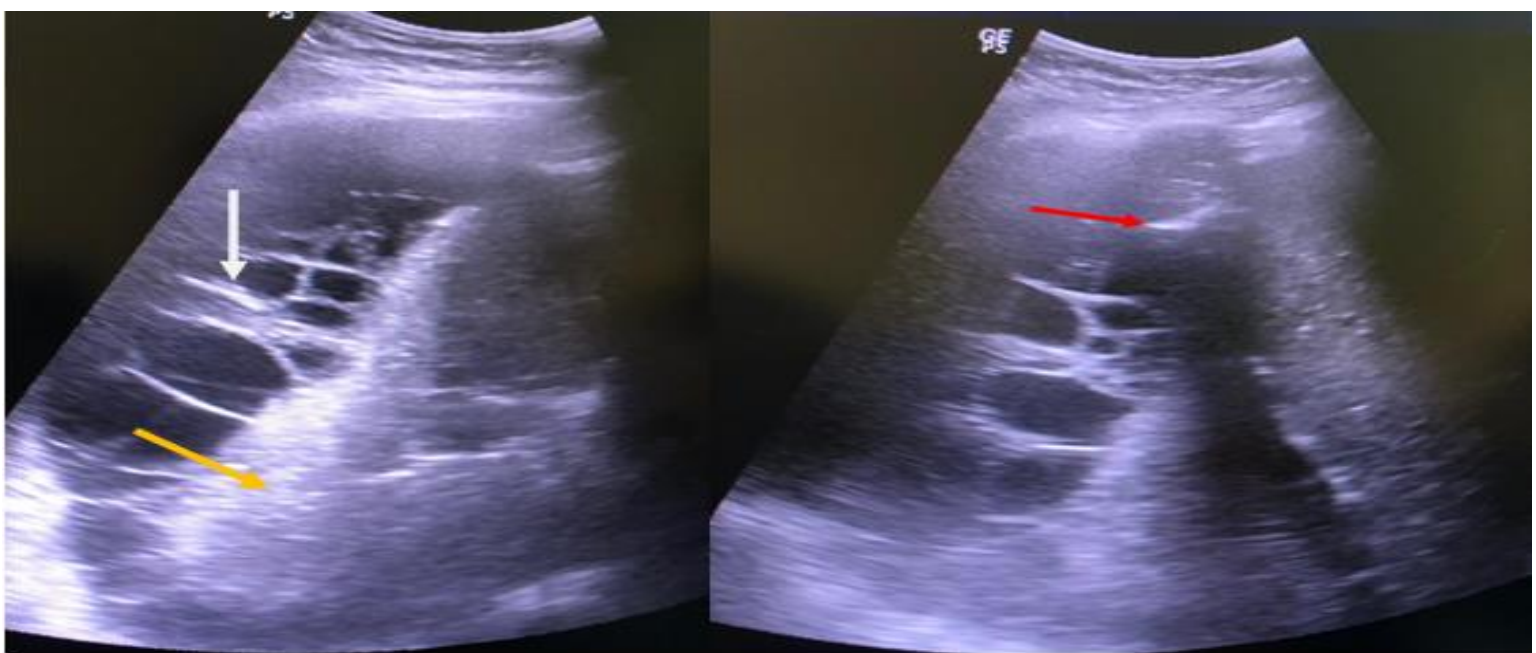

Case (1) Transthoracic ultrasound low frequency convex probe B-mode image showed moderate pleural effusion with septations (white arrow), no pleural thickening with normal adjacent lung (yellow arrow) .thoracentesis needle tip (red arrow) 


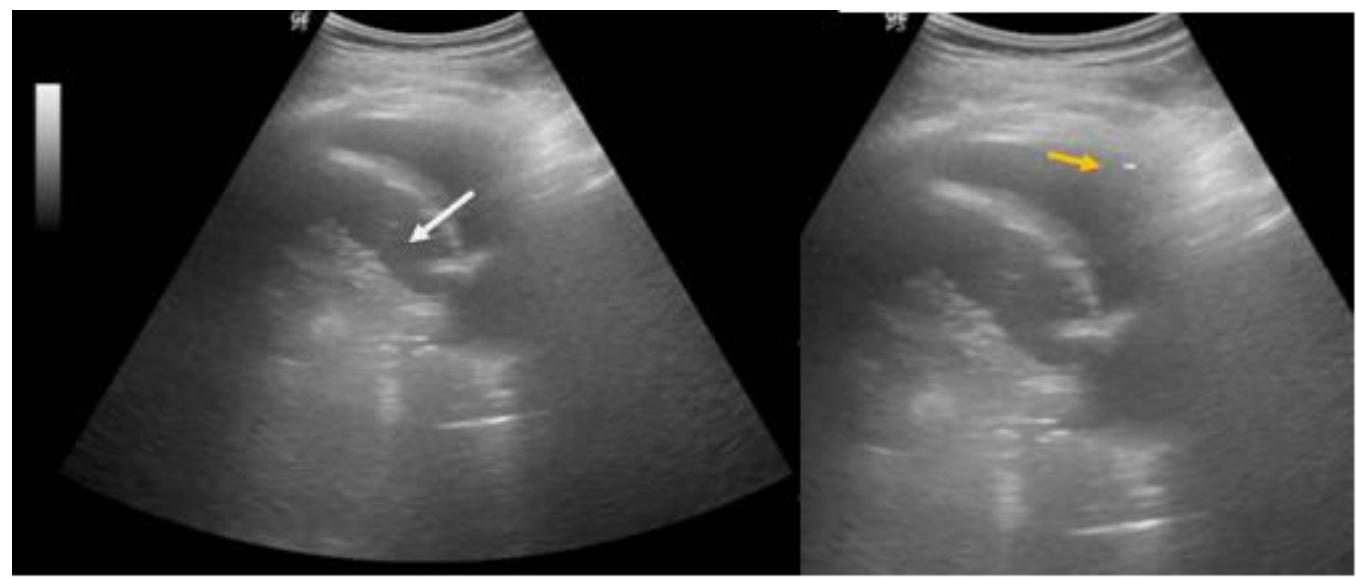

Fig (2) Transthoracic ultrasound low frequency convex probe B-mode image showed mild anechoic pleural effusion ,no septations, no pleural thickening .Normal adjacent lung(white arrow).The needle tip ( yellow arrow) is seen within the effusion.

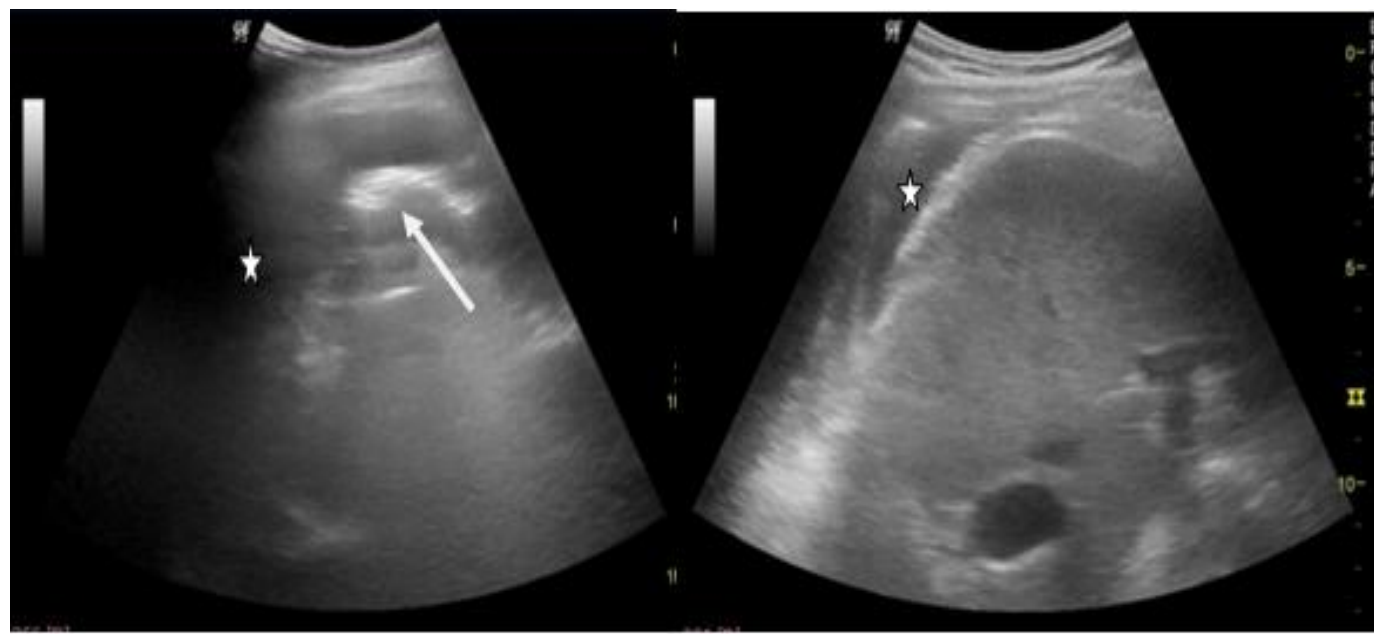

Fig (3) Transthoracic ultrasound low frequency convex probe B-mode image showed minimal anechoic pleural effusion (star), no septations ,no pleural thickening with normal adjacent lung (white arrow) .

\section{Discussion}

Our examination was done on forty patients of which $26(65 \%)$ were guys and $14(35 \%)$ were females while the investigation of Nabil F A, et al.[11] was done on $18(60 \%)$ guys and $12(40 \%)$ females.

As respect side of the radiation, the current investigation demonstrated that (half) had left sided pleural emanation and $(40 \%)$ had right sided pleural emission. while in Kabil ,et al.[12] study (56.67\%) had right sided pleural emanation and $(43.33 \% \%)$ had left sided pleural radiation.

Our investigation uncovered that (25\%) had parapneumonic radiation while Terry L , et al. [13] indicated that $(19 \%)$ had parapneumonic radiation.

Comparable to kind of emanation, the current investigation recognized that $8(20 \%)$ were transudates and $32(80 \%)$ were exudates. While Ramya C. ,et al.[14] found that that $24(30 \%)$ were transudates and $56(70 \%)$ were exudates.

Concerning reasons for emanation, Bugalho A., et al. [15] study was completed on 133 patients (age $67 \pm$
16 years) with the last finding was harmful pleural emission in 66 cases and non-threatening pleural emanation in 67 cases with a general affectability of $80.3 \%$, a particularity of $83.6 \%$, and positive and negative prescient estimations of 82.8 and $81.2 \%$, individually, for the identification of danger. Rather than the current investigation that indicated that 8 cases were dangerous emanation and 32 cases were non threatening pleural radiation with a general affectability of half, a particularity of $87.5 \%$, and positive and negative prescient estimations of 50 and $87.5 \%$, individually, for the recognition of harm.

In diagnosing parapneumonic pleural emission, the current examination indicated an affectability of half, explicitness of $75 \%$, PPV of $33.3 \%$ and NPV of $85.7 \%$ .While the investigation of Ibrahim I.Elmahalawy, et al.[16] demonstrated an affectability of $94 \%$, particularity of $96 \%$, PPV of $97 \%$ and NPV of $90 \%$.

The current examination recognized that $0 \%$ of cases had created pneumothorax following ultrasound guided thoracentesis method while Tonolini 
Massimo[17] expressed that 3\% of cases in his investigation created pneumothorax following the technique.

\section{Conclusion}

TUS is an efficient, quick, inexpensive, radiationfree method for the evaluation of pleural diseases.

\section{References}

[1] R. W. Light, Pleural diseases. Lippincott Williams \& Wilkins, Vol. 1(1), PP. 1-20, 2007.

[2] E. P. Widmaier, H. Raff, K. T. Strang, Vander's Human physiology: the mechanisms of body function. Boston: McGraw-Hill Higher Education, Vol. 2 ( 3), PP. 34-65, 2008.

[3] L. A. Helala, A Madkour, N. M. Osman,. Role of ultrasound in diagnosis and treatment of pleural diseases in respiratory intensive care unit. Egypt J Bronchol, Vol. 9 (1), PP. 79-91, 2015.

[4] S. J. Koenig, M. Narasimhan, P. H. Mayo, "Thoracic ultrasonography for the pulmonary specialist," Chest, Vol. 140 (5) , PP.13321341, 2011.

[5] L. Vetrugno, G. Volpicelli, F. Barbariol,. Phantom model and scoring system to assess ability in ultrasound-guided chest drain positioning. Crit Ultrasound J.,Vol. 8 (1), PP.1, 2016.

[6] P.-C. Yang, K.-T. Luh, D.-B. Chang, "Value of sonography in determining the nature of pleural effusion: analysis of 320 cases.," AJR. Am. J. Roentgenol., Vol. 159, (1), PP. 29-33, 1992.

[7] P. Z. Svigals,A. Chopra , J. G. Ravenel,. The accuracy of pleural ultrasonography in diagnosing complicated parapneumonic pleural effusions. Thorax, Vol. 72, (1), PP. 94-95, 2017.

[8] S. E. Kozmic, D. B. Wayne, J. Feinglass, "Factors associated with inpatient thoracentesis procedure quality at university hospitals," Jt.
Comm. J. Qual. Patient Saf., Vol. 42 (1), PP.3442, 2016

[9] S. Scarlata ,"Estimation of Pleural Effusion Volume by Chest Ultrasound: Validation of Two Multiplanar Models." Eur Respiratory Soc, Vol. 2 (1), PP. 2-32, 2019.

[10] M. H. Faheem, "Is transthoracic ultrasound (TUS) a reliable predictor of the nature of pleural and peripheral pulmonary lesions? Correlation with cyto-histological findings," Egypt. J. Radiol. Nucl. Med., Vol. 50 ,(1), PP.1-8, 2019.

[11] A. K. Reddy, S. B. Kaup, A. E. Jayamohan, , "Sonographic Evaluation of Pleural Effusion," Open J. Med. Imaging, Vol. 7, (3), PP.77-88, 2017.

[12] W. Cao, Y. Wang, N. Zhou, "Efficacy of ultrasound-guided thoracentesis catheter drainage for pleural effusion," Oncol. Lett., Vol. 12, (6) PP. 4445-4448, 2016.

[13] N. A. Maskell,R. J. Butland. BTS guidelines for the investigation of a unilateral pleural effusion in adults. Thorax, Vol. 58, (2), PP.8-17, 2003.

[14]A.N. Rubinowitz, M.D. Siegel, X. TocinoThoracic imaging in the ICU.Crit. Care Clin., Vol. 23 (3), PP. 539-73, 2007.

[15]F. Cortellaro, S. Colombo, D. Coen,. Lung ultrasound is an accurate diagnostic tool for the diagnosis of pneumonia in the emergency department. Emerg. Med. J., Vol. 29 (2), PP.19-23, 2012.

[16] D. R. Duncan,T. I. Morgenthaler, J. H. Ryu,.Reducing iatrogenic risk in thoracocentesis. Chest, Vol. 135, (3), PP. 13151320, 2009.

[17] G. Volpicelli, M. Elbarbary, M. Blaivas,. "International evidence based recommendations for point of care lung ultrasound," Intensive Care Medicine, Vol. 38 (4), PP.577-591, 2012. 